\title{
Article
}

\section{Free energies of hydrated halide anions: High through-put computations on clusters to treat rough energy-landscapes}

\author{
Diego T. Gomez ${ }^{1, \ddagger}$, Lawrence R. Pratt ${ }^{2}$, , David M. Rogers 3 3,, and Susan B. Rempe ${ }^{4, \ddagger} *$ \\ Engineering, Tulane University; dgomez1@tulane.edu \\ 2 Department of Chemical \& Biomolecular Engineering, Tulane University; lpratt@tulane.edu \\ 3 National Center for Computational Sciences, Oak Ridge National Laboratory; rogersdm@ornl.gov \\ 4 Center for Integrated Nanotechnologies, Sandia National Laboratories; slrempe@sandia.gov \\ * Correspondence: slrempe@sandia.gov; Tel.: +1-505-845-0253 (S.R.) \\ $\ddagger$ These authors contributed equally to this work.
}

\begin{abstract}
With a longer-term goal of addressing the comparative behavior of the aqueous halides $\mathrm{F}^{-}, \mathrm{Cl}^{-}, \mathrm{Br}^{-}$, and $\mathrm{I}^{-}$on the basis of quasi-chemical theory (QCT), here we study structures and free energies of hydration clusters for those anions. We confirm that energetically optimal $\left(\mathrm{H}_{2} \mathrm{O}\right)_{n} \mathrm{X}$ clusters, with $\mathrm{X}=\mathrm{Cl}^{-}, \mathrm{Br}^{-}$, and $\mathrm{I}^{-}$, exhibit surface hydration structures. Computed free energies based on optimized surface hydration structures utilizing a harmonic approximation, typically (but not always) disagree with experimental free energies. To remedy the harmonic approximation, we utilize single-point electronic structure calculations on cluster geometries sampled from an AIMD (ab initio molecular dynamics) simulation stream. This rough-landscape procedure is broadly satisfactory and suggests unfavorable ligand crowding as the physical effect corrected. Nevertheless, this procedure can break down when $n \gtrsim 4$, with the characteristic discrepancy resulting from a relaxed definition of clustering in the identification of $\left(\mathrm{H}_{2} \mathrm{O}\right)_{n} \mathrm{X}$ clusters, including ramified structures natural in physical cluster theories. With ramified structures, the central equation for the present rough-landscape approach can acquire some inconsistency. Extension of these physical cluster theories in the direction of QCT should remedy that issue, and should be the next step in this research direction.
\end{abstract}

Keywords: ion hydration, physical cluster theory, halides, Hofmeister series, specific ion effects

\section{Introduction}

Ions exert specific effects on molecules. Hofmeister devised a ranked list of salts to classify their influence on protein precipitation and swelling [1,2]. Later works identified ion-specific effects on molecules more generally, in both aqueous and non-aqueous solutions, at interfaces, and in confined settings such as biological transport proteins and ionomers [3-6] with little or no solvent. Indeed, molecular-level mechanisms of ion-specific effects is a topic of current research [7-9]. Important aspects of those mechanisms in aqueous solution involves the local ion hydration properties, and exchange of hydrating water molecules for molecular ligating groups [3,5,10-12]. As a step toward enhancing our understanding of ion-specific effects, this paper studies the structures and free energies of hydration clusters of the anions $\mathrm{F}^{-}, \mathrm{Cl}^{-}, \mathrm{Br}^{-}$, and $\mathrm{I}^{-}$in the dilute gas-phase, with the longer term goal of addressing the comparative behavior of this series of ions in liquid water on the basis of quasi-chemical theory (QCT) [12-15]. Accurate characterization of gas-phase clusters can indeed helpfully inform QCT applications, and has been successful for cations [12]. Because our analyses here will be limited to gas phase systems, we restrict our work to traditional physical cluster theories [16-22], progenitors of molecular QCT [23,24].

Common to all these theories is the study of associative equilibria

$$
n \mathrm{~W}+\mathrm{X} \rightleftharpoons \mathrm{W}_{n} \mathrm{X} .
$$


Here $\mathrm{X} \equiv \mathrm{F}^{-}, \mathrm{Cl}^{-}, \mathrm{Br}^{-}$, or $\mathrm{I}^{-}$, and the dissolving medium provides ligand $\mathrm{W} \equiv \mathrm{H}_{2} \mathrm{O}$ molecules. Eq. (1) then directs attention to

$$
K_{n}^{(0)}=\frac{\rho_{\mathrm{W}_{n} \mathrm{X}}}{\rho_{\mathrm{W}} \rho_{\mathrm{X}}}
$$

where $\rho_{\mathrm{W}_{n} \mathrm{X}}$ is the number density of $\mathrm{W}_{n} \mathrm{X}$ species. Then $K_{n}^{(0)}$ is the traditional equilibrium constant appropriate for the case that the dissolving medium is an ideal gas. That ideal gas restriction is indicated by the superscript notation. In QCT, slightly more subtle considerations arrive also at Eq. (2), which here serves as our starting point [25]. These theories all require logical definition of formed $\mathrm{W}_{n} \mathrm{X}$ clusters for counting. Such definitions amount to defining proximity of a $\mathrm{W}$ ligand to an $\mathrm{X}$ ion. Although judgement might be required to establish a physically effective proximity definition, here we will assume that, under the standard temperatures considered below and the low density conditions of interest, practical molecular simulations of $\mathrm{W}_{n} \mathrm{X}$ systems encounter clustered configurations entirely. This definition will be reconsidered in subsequent QCT developments.

The computational study of clusters benefits from a good experimental basis [26]. Widely available chemical software enables straightforward numerical evaluation of $K_{n}^{(0)}$ [26] under assumptions of harmonic motion on the potential energy surface near an optimized cluster geometry (Figure 1). The assumption of harmonic motion is valid for clusters with strong interactions that limit atomic displacements to small distances away from an optimized geometry [27]. A challenge of the harmonic approximation is that there is no guarantee that only one low energy structure is relevant $[28,29]$. Similar to earlier results found for simple cations [30,31], harmonic approximations (Figure 1) are satisfactory for $\mathrm{W}_{n} \mathrm{~F}^{-}$and $n \leq 4$, but not for $n=5$ in that case. From the disagreement with experiment (Figure 1), we see that the harmonic treatment is not satisfactory for $\mathrm{W}_{n} \mathrm{Cl}^{-}$. For those challenging cases, previous work provided a simple fix of that discrepancy [15], making use of cluster configurations sampled from dynamical simulations. The goal of this paper is to test whether inaccuracies of the harmonic treatment are satisfactorily resolved also for the rest of the halide series $\left(\mathrm{Br}^{-}, \mathrm{I}^{-}\right)$by analysis of structures from dynamical simulations. A similar approach was applied earlier to correct harmonic approximations applied to compute chemical equilibria and free energies of $\mathrm{H}_{2}$ hydration clusters [32].

The essential feature of the computational approach here is the deployment of standard electronic structure computations for cluster geometries sampled from AIMD ( $a b$ initio molecular dynamics) simulations of $\mathrm{W}_{n} \mathrm{X}$ clusters. A detailed accounting from the single-point energy procedures defined with Eq. (5) below indicates more than $10^{3}$ calculations for $n=5$ with standard modern electronic structure methods for single geometries from the AIMD canonical simulation stream. Thus, we implement a high-throughput algorithm, described below, to carry-out the calculations efficiently.

We emphasize the potential utility, in the longer term, of this work to QCT applications to bulk solutions. QCT puts a high premium on understanding the structure and energetics of small clusters that can serve to fill-out an inner shell, then treating $\mathrm{W}_{n} \mathrm{X}$ as a primitive chemical constituent of the solution. Therefore, we limit $n$ to values smaller than the historical interest [33-43]. Specifically, we do not attempt to push $n$ to values large enough to suggest an incipient droplet of liquid. Instead, we seek a sharper understanding of $\mathrm{W}_{n} \mathrm{X}$ structures, energetics, and particularly rough landscape effects for small values of $n$ [15]. Nevertheless, the present results are broadly consistent with preceding simulation work on such systems, including on larger values of $n$, studied with empirical intermolecular force fields [33-43]. Though this work purposely avoids taking the large cluster limit, an alternative QCT model which does seek that limit appeared recently [44]. 

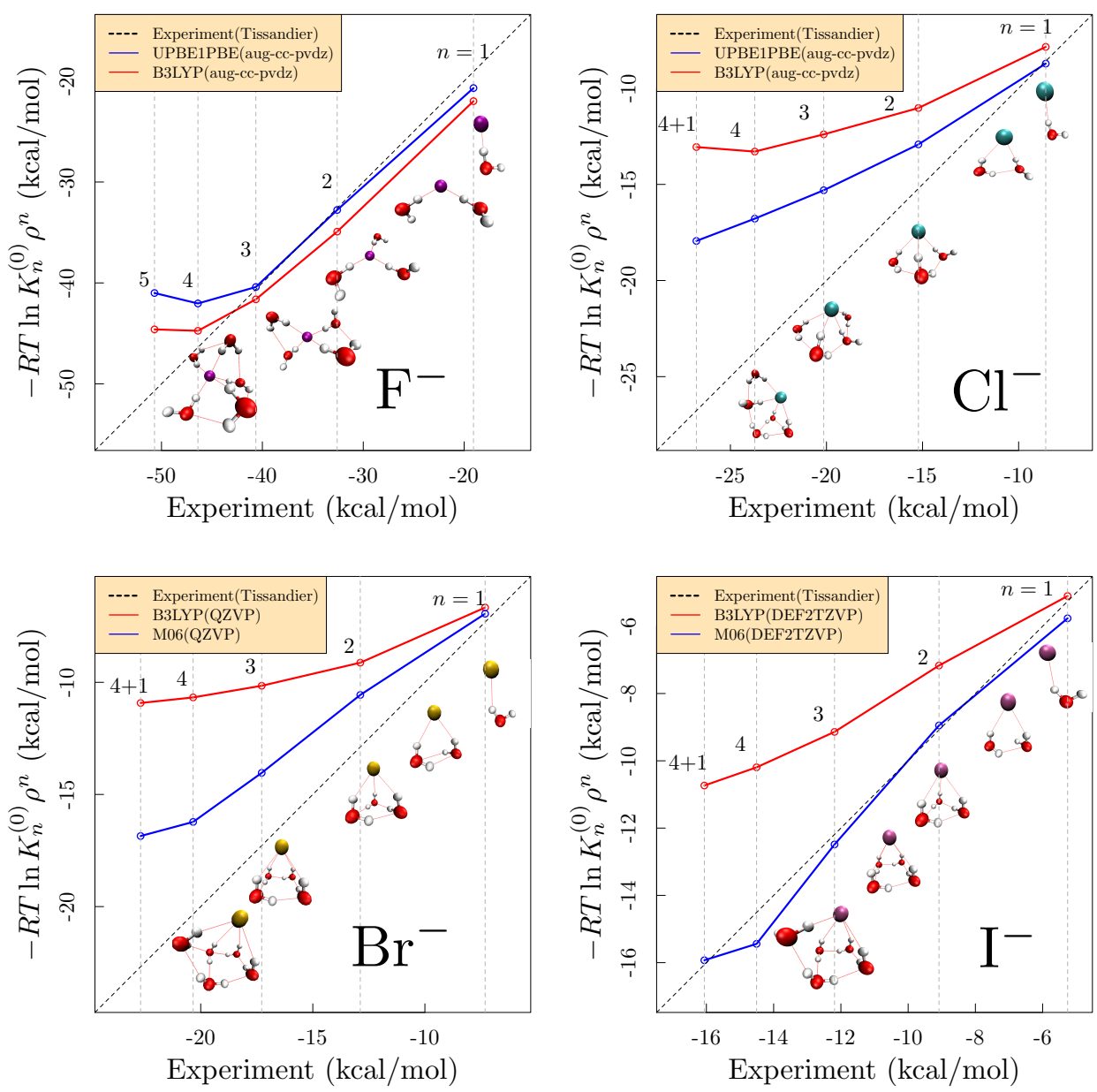

Figure 1. Cluster free energies using harmonic approximation compared with experimental values for $\left(\mathrm{H}_{2} \mathrm{O}\right)_{n} \mathrm{X}, \mathrm{X}=\mathrm{F}^{-} \mathrm{Cl}^{-}, \mathrm{Br}^{-}$, and $\mathrm{I}^{-}$and $1 \leq n \leq 5$. The optimized cluster structures are obtained and analyzed with the Gaussian package, as discussed in Sec. 4.1. The legends indicate electron density functional and basis sets. The inset molecular graphics illustrate that asymmetric, surface-hydrated structures are optimal geometries for clusters involving $\mathrm{Cl}^{-}$and heavier halides. For $n=5$ and $\mathrm{Cl}^{-}, \mathrm{Br}^{-}$, and $\mathrm{I}^{-}$, optimizations pushed a water molecule into a distinct outer shell, and those structures are denoted as $4+1$ clusters. The free energy evaluated using the harmonic approximation for $\mathrm{Cl}^{-}$and $\mathrm{Br}^{-}$deviates from experiment and the deviations grow larger with $n$. For these ions, the harmonic approximation for the $n=1$ case produces an accurate estimate for the experiment, which indicates the dissimilarity for the other cluster sizes $n \geq 2$ arises from interactions between the waters. 


\section{Results}

We evaluated structures and formation free energies of $W_{n} X$ clusters in gas phase. We compared: i) the experimental values determined from mass spectrometry [45]: ii) the quantum-mechanical rigid rotor harmonic oscillator approximation (Figure 1), and iii) the rough-energy landscape procedure proposed in Ref [15]. Figure 2 summarizes all results graphically. The free energies decrease by about $5 \mathrm{kcal} / \mathrm{mol}$ for each water added. Free energies follow the expected size trend - smaller anions bind water more strongly. Associated with the energetic trend, and correlated with increasing anion polarizability [46], the first solvation shell becomes more asymmetric with increasing anion radius.

For the $\mathrm{Cl}^{-}$and $\mathrm{Br}^{-}$ions (Figure 2), the rough-landscape procedure (Eq. (6)) makes the distinctive correction anticipated except for the $n \gtrsim 4$ cases. That exceptional $n \gtrsim 4$ behavior is exhibited also by $\mathrm{I}^{-}$, though the $\mathrm{I}^{-}$case is unusual in that the harmonic approximation is accurate. We attribute the exceptional $n \gtrsim 4$ behavior to the influence of split-shell structures, including $4+1$ structures for $n=5$, in the data stream for these physical clusters. Split-shell clusters have been documented for simple cations [48-53], and used to rationalize discrepancies between computed and experimental gas phase cluster data [54]. The $\mathrm{F}^{-}$case is simpler overall, though $n \geq 4$ begins to incur the characteristic error of the harmonic approximation in this application. Still the roughlandscape procedure (Eq. (6)) improves the comparison with experiment for the $\mathrm{F}^{-}$case, too.

Structures of the $W_{5} X$ clusters support these observations about split-shell structures (Figure 3): $\mathrm{F}^{-}$is ideally simple, and other cases are individually more complicated. These points hold also for the $\mathrm{W}_{3} \mathrm{X}$ clusters (Figure 4), which might have been guessed to be significantly simpler.

\section{Discussion}

We confirm that energetically optimal $\left(\mathrm{H}_{2} \mathrm{O}\right)_{n} \mathrm{X}$ clusters with $\mathrm{X}=\mathrm{Cl}^{-}, \mathrm{Br}^{-}$, and $\mathrm{I}^{-}$exhibit surface hydration structures. For those surface hydration cases, free energies based on harmonic approximation of the potential energy surface and optimal structures typically (but not always) disagree with experimental free energies. The rough-landscape remedy to the harmonic approximation accounts for unfavorable effects (Figure 5) due to crowding of the ligands.

The tested rough-landscape procedure provides a distinctive correction, but breaks down in the present implementation in comparison with experiment when $n \gtrsim 4$. The characteristic discrepancy for $n \gtrsim 4$ is suggested to be a consequence of a relaxed definition of clustering in the identification of $\left(\mathrm{H}_{2} \mathrm{O}\right)_{n} \mathrm{X}$ clusters - including split-shell or ramified structures - that accompany physical cluster theories. With ramified structures, the central equation (Eq. (6)) for the present rough-landscape approach can acquire some inconsistency. Further development of these physical cluster theories in the direction of QCT should remedy this issue, and should be the next step in this research direction.

\section{Materials and Methods}

\subsection{Software and Procedures}

Molecular dynamics trajectories of the isolated $\left(\mathrm{H}_{2} \mathrm{O}\right)_{n} \mathrm{X}$ for $2 \leq n \leq 5$ and $\mathrm{X}=\mathrm{F}^{-}$ $\mathrm{Cl}^{-}, \mathrm{Br}^{-}$, and $\mathrm{I}^{-}$were obtained using CP2K [55,56]. The M06 [57] functional was utilized as our standard case with pseudopotentials proposed by Goedecker, Teter and Hutter (GTH [58]) in the Gaussian and plane wave schemes [59]. Molecularly optimized DZVPMOLOPT-SR-GTH [60] basis sets were obtained from the CP2K website. Temperatures were set at $300 \mathrm{~K}$ with the Nosé-Hoover thermostat, a time step of $1 \mathrm{fs}$ for 15 ps total of trajectory with the last 10 ps used for analysis.

Our initial assessment of cluster free energies (Figure 1) applied the harmonic approximation to geometry-optimized structures. As initial conditions for the geometry optimization, $N_{s}=20$ uniformly spaced configurations were extracted from the last 10 ps of $\mathrm{CP} 2 \mathrm{~K}$ trajectories and those sampled configurations were optimized using Gaussian09 

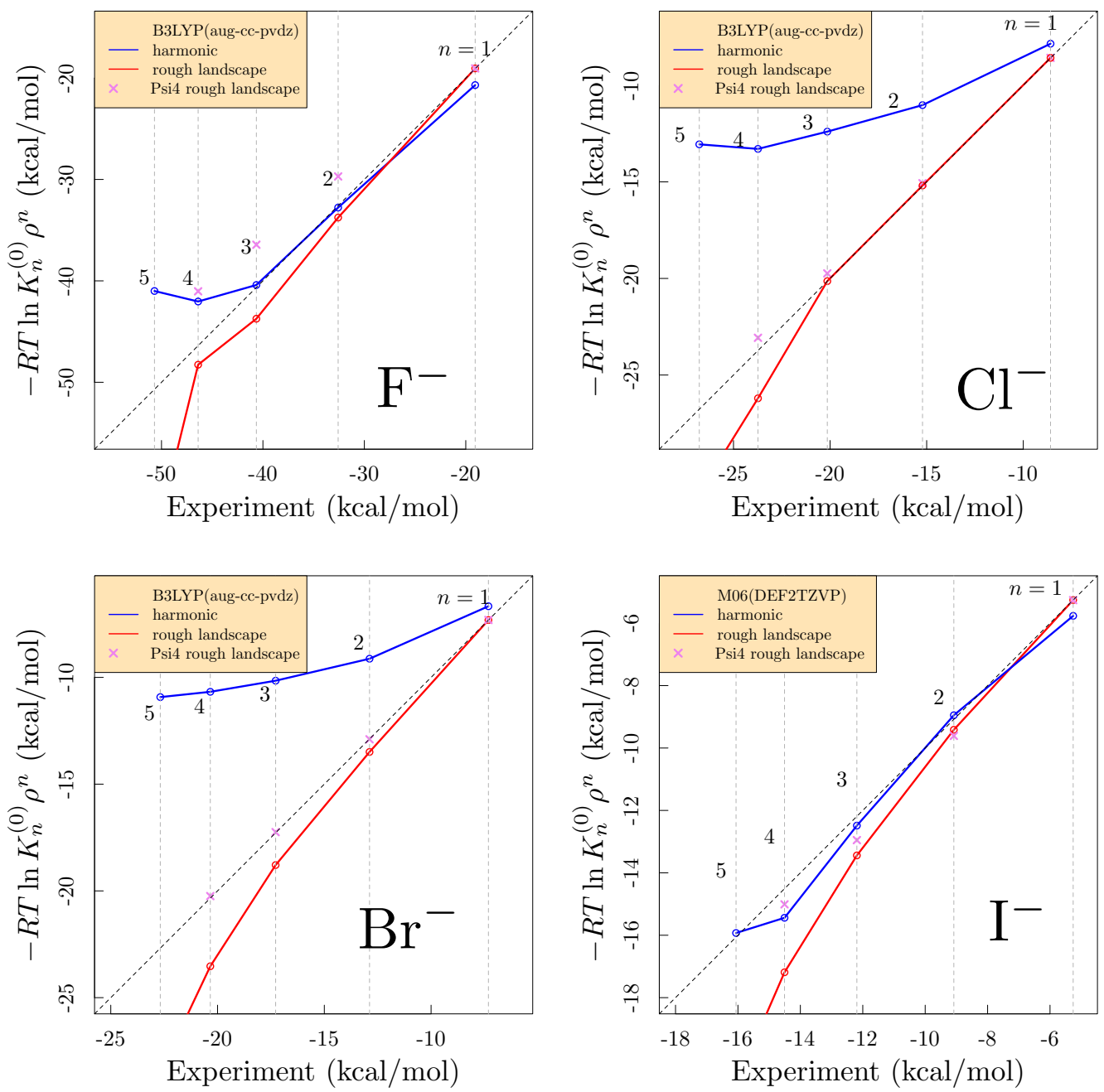

Figure 2. Harmonic (blue) and rough landscape (red) free energies compared to experimental results (dashed line [45]). For $n=1,2,3$ clusters, expected to be solely inner-shell, electronic structure sampling of the rough landscape yields results that agree well with experiment. As cluster size increases, some waters occupy an outer solvation shell, at least intermittently. That split-shell occupancy reduces repulsive ligand crowding (Figure 5), and the clustering consistency of Eq. (6) might deteriorate. For comparison, the crosses show the rough landscape analysis utilizing the PBE functional [47], both for the CP2K trajectory and the electronic structure calculations using Psi4 for analysis. For that case, structures sampled from the trajectory are more compact, and the accuracy of the rough landscape approach seems slightly better. 

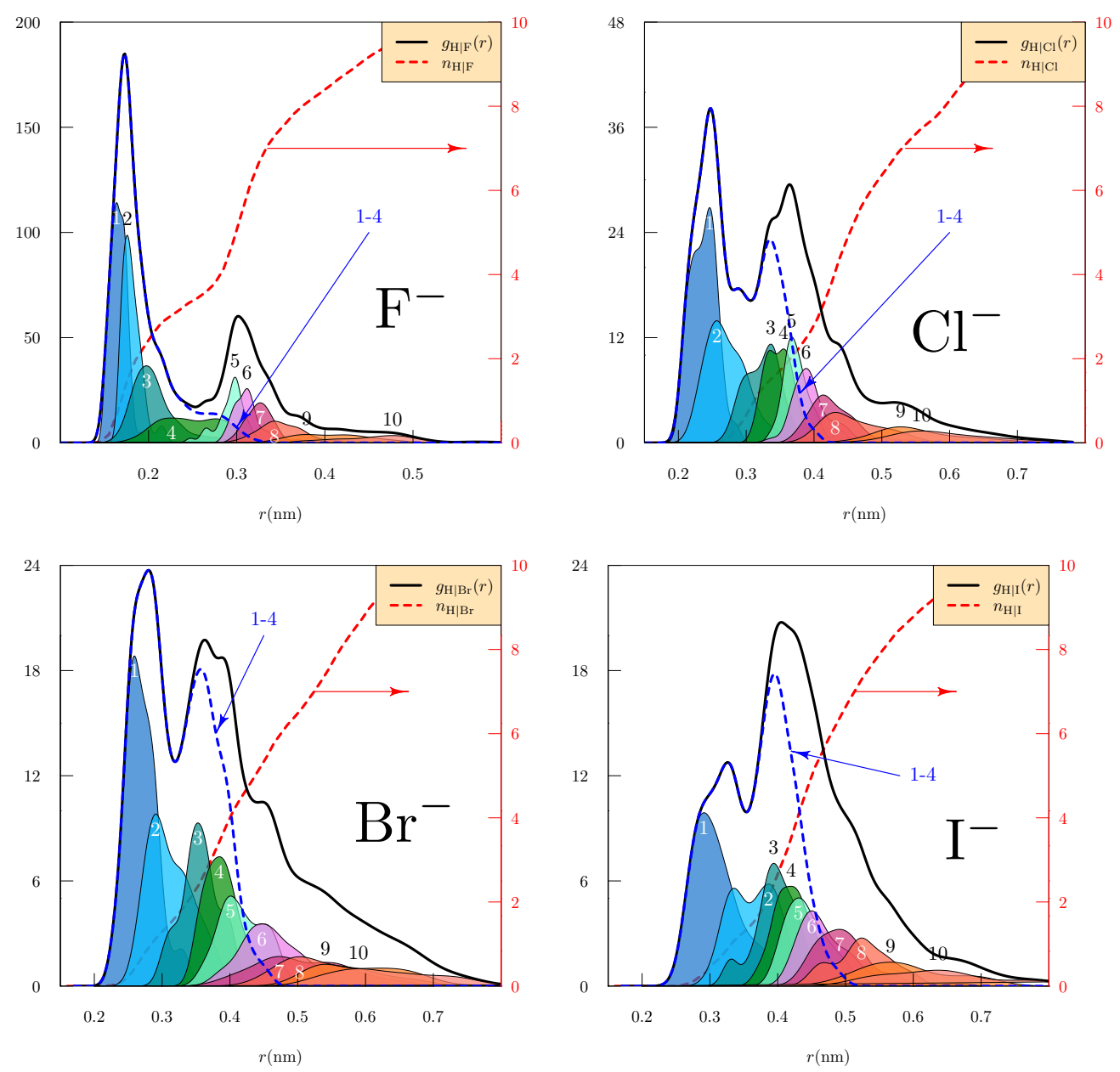

Figure 3. Radial distributions of $\mathrm{H}$ atoms from the indicated ions observed from 5 ps of AIMD trajectory of the clusters $W_{5} X$, after 5 ps of aging as discussed in Sec. 4 . The integer labeled curves are distributions of neighborship-ordered $\mathrm{H}$ atoms, e.g., the curve labeled ' 1 ' is the distribution of the nearest $\mathrm{H}$ atom. The distributions and the vertical axes have units of $1 / \mathrm{nm}^{3}$, and those distributions are normalized as $4 \pi \int_{0}^{\infty} \rho^{(j)}(r) r^{2} \mathrm{~d} r=1$ The average included number of $\mathrm{H}$-atoms, $n_{\mathrm{H} \mid \mathrm{X}}(r)$ is the red-dashed curves and right-side axes. $n_{\mathrm{H} \mid \mathrm{X}}(r)$ for the $\mathrm{F}^{-}$case has a distinct step near $n_{\mathrm{H} \mid \mathrm{X}}(r)=4$, indicating classic 4-coordinate structure with $\mathrm{H}$-bond donation for that innershell. For the $\mathrm{F}^{-}$case, the sum of the distributions 1-4 accurately fills out the net distribution for $r \lesssim 0.25 \mathrm{~nm}$. 
[61] with the B3LYP $[62,63]$ functional for all ions, in addition to PBE $[64,65]$ for $\mathrm{F}^{-}$and $\mathrm{Cl}^{-}$utilizing the aug-cc-pvdz basis set [66,67], and M06 for $\mathrm{Br}^{-}$and $\mathrm{I}^{-}$utilizing QZVP [68] and DEF2TZVP [69] basis sets, respectively. Finally, $-R T \ln K_{n}^{(0)}$ (Figure 1) was evaluated in the harmonic approximation for the lowest energy structure. The symmetry number - the $n$ ! of Eq. (3) — was assigned as discussed by Muralidharan, et al., [70]. For $\left(\mathrm{H}_{2} \mathrm{O}\right)_{5} \mathrm{Br}^{-},\left(\mathrm{H}_{2} \mathrm{O}\right)_{5} \mathrm{Cl}^{-}$and $\left(\mathrm{H}_{2} \mathrm{O}\right)_{5} \mathrm{I}^{-}$(Figure 1), optimizations push a water molecule into an outer solvation shell, and those structures thus can be identified as $4+1$ clusters.

For the rough landscape treatment, structures from $2 \leq n \leq 5$ clusters are sampled every 0.1 ps of the last 10 ps of the AIMD trajectory. Each sampled configuration is decoupled, according to the right-hand side of Eq. (5), and the structures subjected to single-point calculations using Gaussian09 with the B3LYP functional and aug-cc-pvdz basis set for F, Cl, Br and M06 functional and DEF2TZV basis set for I configurations. Using the resulting thermal averaging in Eq. (6), and $K_{1}^{(0)}$ from experiment, the resulting $K_{n}^{(0)}$ produce the results in Figure 1 in a step-wise fashion.

To confirm our results, and to investigate the functional dependence of the cluster free energy, additional AIMD trajectories for $2 \leq n \leq 5$ clusters of all halides were carried out independently. These were also done with CP2K, this time using the PBE [64] functional with GTH psuedopotentials and molecularly optimized DZVP-MOLOPT-SRGTH basis set, using the Nosé-Hoover thermostat at $300 \mathrm{~K}$. Structures from AIMD were again sampled every $0.1 \mathrm{ps}$ from the last $10 \mathrm{ps}$ of $15 \mathrm{ps}$ of the CP2K trajectory. Single-point energy computations of the energy differences indicated by Eq. 5 for $2 \leq n \leq 5$ clusters, used the PBE functional and all-electron DEF2TZVPD [71] basis set, as implemented by Psi4 [72].

\subsection{Theory}

Classic statistical thermodynamics $[13,15,73]$ anchors our analysis of

$$
K_{n}^{(0)}=\frac{\mathcal{Q}\left(\mathrm{W}_{n} \mathrm{X}\right) / n !}{\mathcal{Q}(\mathrm{X})[\mathcal{Q}(\mathrm{W}) / V]^{n}} .
$$

Here $V$ is the system volume and $\mathcal{Q}\left(\mathrm{W}_{n} \mathrm{X}\right)$ are single molecule (cluster) canonical partition functions, configurational integrals in the traditional classical-limit analysis [73]. In this ratio, symmetry numbers associated with $\mathrm{W}$ molecules can be cancelled on topand-bottom, and the $n$ ! reflects the permutation symmetry of the $n \mathrm{~W}$ ligands. Each $\mathcal{Q}\left(\mathrm{W}_{n} \mathrm{X}\right)$ is proportional to $V$, so the ratio Eq. (3) is independent of $V$.

Our scheme for evaluating $K_{n}{ }^{(0)}$ proceeds by step-wise addition of waters $(n-1 \rightarrow$ $n$ ) according to

$$
\frac{K_{n}{ }^{(0)}}{K_{n-1}{ }^{(0)} K_{1}{ }^{(0)}}=\frac{\mathcal{Q}\left(\mathrm{W}_{n} \mathrm{X}\right) / \mathcal{Q}(\mathrm{X})}{n\left[\mathcal{Q}\left(\mathrm{W}_{n-1} \mathrm{X}\right) / \mathcal{Q}(\mathrm{X})\right][\mathcal{Q}(\mathrm{WX}) / \mathcal{Q}(\mathrm{X})]} .
$$

The numerator on the right of Eq. (4), involves integration carried over configurations of a $\mathrm{W}_{n} \mathrm{X}$ cluster, i.e., integrated over clustered configurations, canonically weighted as $\exp \left[-\beta U\left(\mathrm{~W}_{n} \mathrm{X}\right)\right]$. Here $\beta=1 / k T$, and $U\left(\mathrm{~W}_{n} \mathrm{X}\right)$ is the electronic energy of the $\mathrm{W}_{n} \mathrm{X}$ cluster in a given geometry. Because of the divisor in the combination $\mathcal{Q}\left(\mathrm{W}_{n} \mathrm{X}\right) / \mathcal{Q}(\mathrm{X})$, that combination is independent of the system volume. The combined denominator treats configurations of $\mathrm{W}_{n-1} \mathrm{X}$ and $\mathrm{WX}$, but independently of each other. Of course, exactly the same integrations are expressed in numerator and denominator of Eq. (4). Introducing

$$
\Delta U_{n}=U\left(\mathrm{~W}_{n} \mathrm{X}\right)-U\left(\mathrm{~W}_{n-1} \mathrm{X}\right)-U(\mathrm{WX})+U(\mathrm{X}),
$$


then the denominator of Eq. (4), expressing the same integrations as the numerator, is merely missing the factor $\exp \left[\beta \Delta U_{n}\right]$. Therefore

$$
n K_{n}{ }^{(0)}=\frac{K_{1}{ }^{(0)} K_{n-1}{ }^{(0)}}{\left\langle\mathrm{e}^{\beta \Delta U_{n}}\right\rangle_{n}} .
$$

The brackets, $\langle\ldots\rangle_{n}$, indicate the thermal average utilizing configurations from the canonical simulation stream for the $\mathrm{W}_{n} \mathrm{X}$ cluster [15]. Here clustering is assessed in the same way on top and bottom: a clustered $\mathrm{W}_{n} \mathrm{X}$ configuration is analyzed as clustered configurations of $\mathrm{W}_{n-1} \mathrm{X}$ and $\mathrm{WX}$. This amounts to an approximation if the $\mathrm{W}_{n} \mathrm{X}$ clusters are frequently ramified, i.e., branched. To see that point, suppose that the singlet $\mathrm{W}$ identified for Eq. (5) is a node of the connectivity graph. Then the $W_{n-1} X$ remainder would not be connected. This concern is relieved for the more compact clusters of QCT applications, in contrast to the "Stillinger clusters" [19] for the physical cluster theory followed here.

In the energy combination of Eq. (5), the rightmost term depends on the geometry of the $\mathrm{W}_{n} \mathrm{X}$ cluster sampled. The energies following that rightmost term are evaluated for a given conformation of $\mathrm{W}_{n} \mathrm{X}$ from the simulation stream for that cluster. One ligand (or each in turn) is distinguished to compose the energy difference suggested by the exchange

$$
\mathrm{WX}+\mathrm{W}_{n-1} \mathrm{X} \rightleftharpoons \mathrm{X}+\mathrm{W}_{n} \mathrm{X} .
$$

Geometries of both species on the left of Eq. (7) conform to the sampled $W_{n} X$ structure on the right. One understanding of $\Delta U_{n}$ (Eq. (5)) is based on the following accounting. Consider first the combination $[U(\mathrm{WX})-U(\mathrm{X})]$ on the right side of Eq. (5). This is the energy change for introducing one $W$ ligand to a bare $X$ ion. Next consider the remaining contribution $U\left(\mathrm{~W}_{n} \mathrm{X}\right)-U\left(\mathrm{~W}_{n-1} \mathrm{X}\right)$. This is the energy change for introducing an additional $\mathrm{W}$ ligand to a $\mathrm{W}_{n-1} \mathrm{X}$ complex. The difference $\Delta U_{n}$ thus reflects the crowding of the $n^{\text {th }} \mathrm{W}$ ligand, including any effect of suboptimal binding of the $n^{\text {th }} \mathrm{W}$ ligand to the $\mathrm{X}$ ion.

The free energies we arrive at correspond to water addition reactions (Eq. (1)). They are experimentally measured by creating an ensemble with varying number of waters $(n)$ in gas-phase mass spectrometry.[45] Because the cluster's net charge remains constant as $n$ varies, the energy combination of Eq. (5) is not affected by the electrostatic potential of the phase [12].

For $n=1$, Eq. (6) correctly reduces to the trivial case of $K_{0}{ }^{(0)}=1$. In the evaluation of $K_{n}{ }^{(0)}$ for $n \geq 2$, the value of $K_{1}{ }^{(0)}$ can be supplied from experiment [45] or theory. This term incorporates the interaction strength between $X$ and one $W$ molecule. Carrying out subsequent steps in this scheme then addresses the issues that make anion hydration more challenging, i.e., competing H-bonding interactions of neighboring $\mathrm{W}$ molecules in those clusters.

The analysis above is based on the classical limit formula for the partition function $\mathcal{Q}\left(\mathrm{W}_{n} \mathrm{X}\right)$. Thus, this approach does not directly address issues of quantum mechanical zero-point motion, except to the extent that a pragmatic external evaluation of $K_{1}^{(0)}$ incorporates zero-point motion empirically. This treatment of zero-point motion differs from the preceding harmonic approximation.

\section{Conclusions}

The rough-landscape analysis (Eq. (6)) is surprisingly accurate compared to the available experiment data (Figure 2), even for the cases of ions that exhibit qualitative, though characteristic, anharmonic behaviors (Figure 1).

Funding: The work of DTG and LRP was supported in part by by the Laboratory Directed Research and Development (LDRD) Program at Los Alamos National Laboratory under project number 20190057DR. The work of SLR was support in part by the LDRD Program at Sandia National Laboratories. This research also used resources provided by the Los Alamos National 

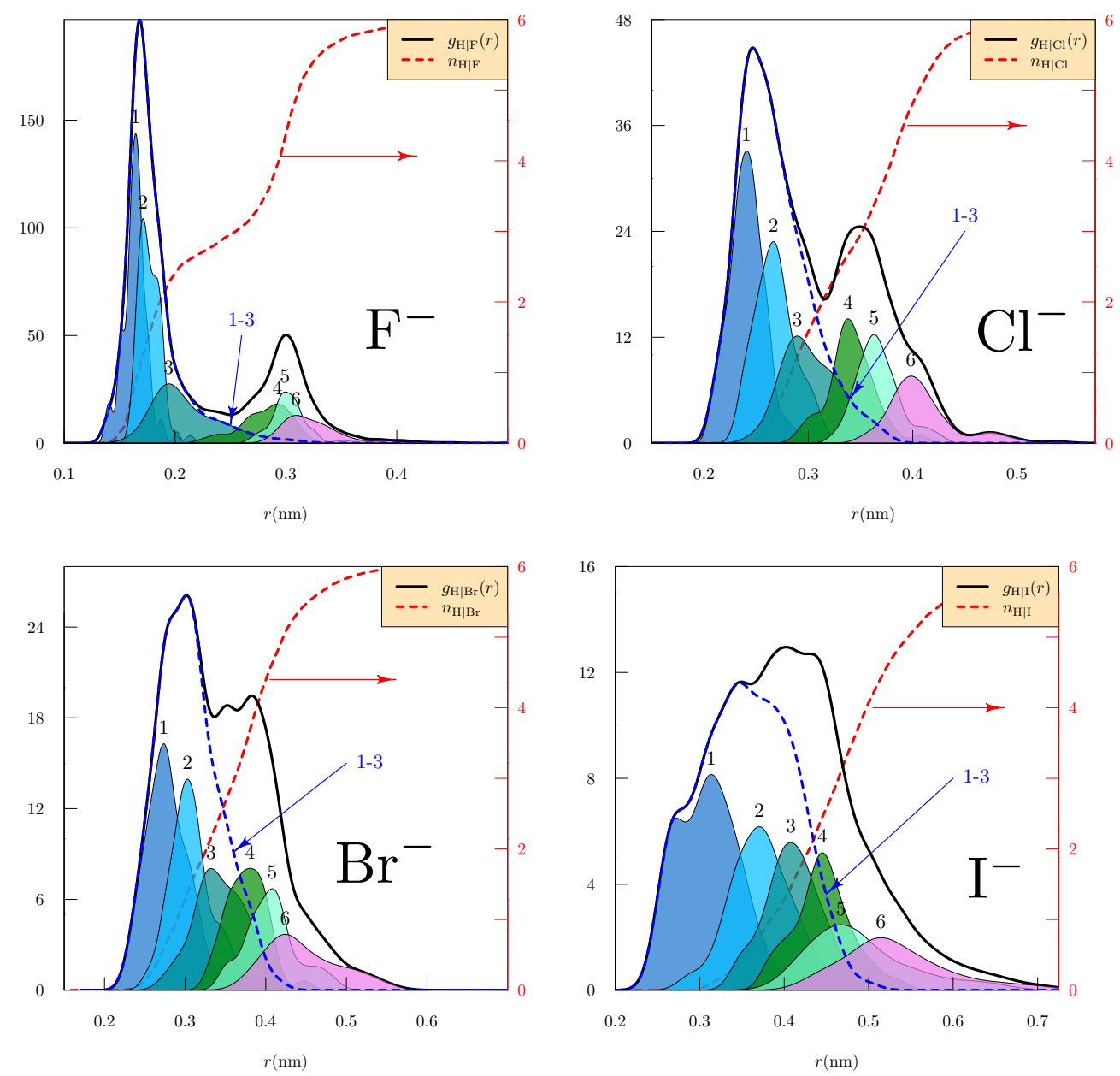

Figure 4. Radial distributions of $\mathrm{H}$ atoms from the indicated ions observed from 5 ps of AIMD trajectory of the clusters $W_{3} X$ after 5 ps of aging, as discussed in Sec. 4 . The distributions and the vertical axes have units of $1 / \mathrm{nm}^{3}$. The integer labeled curves are distributions of neighborshipordered $\mathrm{H}$ atoms, as discussed in Figure 3, and normalized as $4 \pi \int_{0}^{\infty} \rho^{(j)}(r) r^{2} \mathrm{~d} r=1$. The average included number of $\mathrm{H}$-atoms $\left(n_{\mathrm{H} \mid \mathrm{X}}(r)\right.$, red-dashed curve), which are gauged by the right-side axes. 

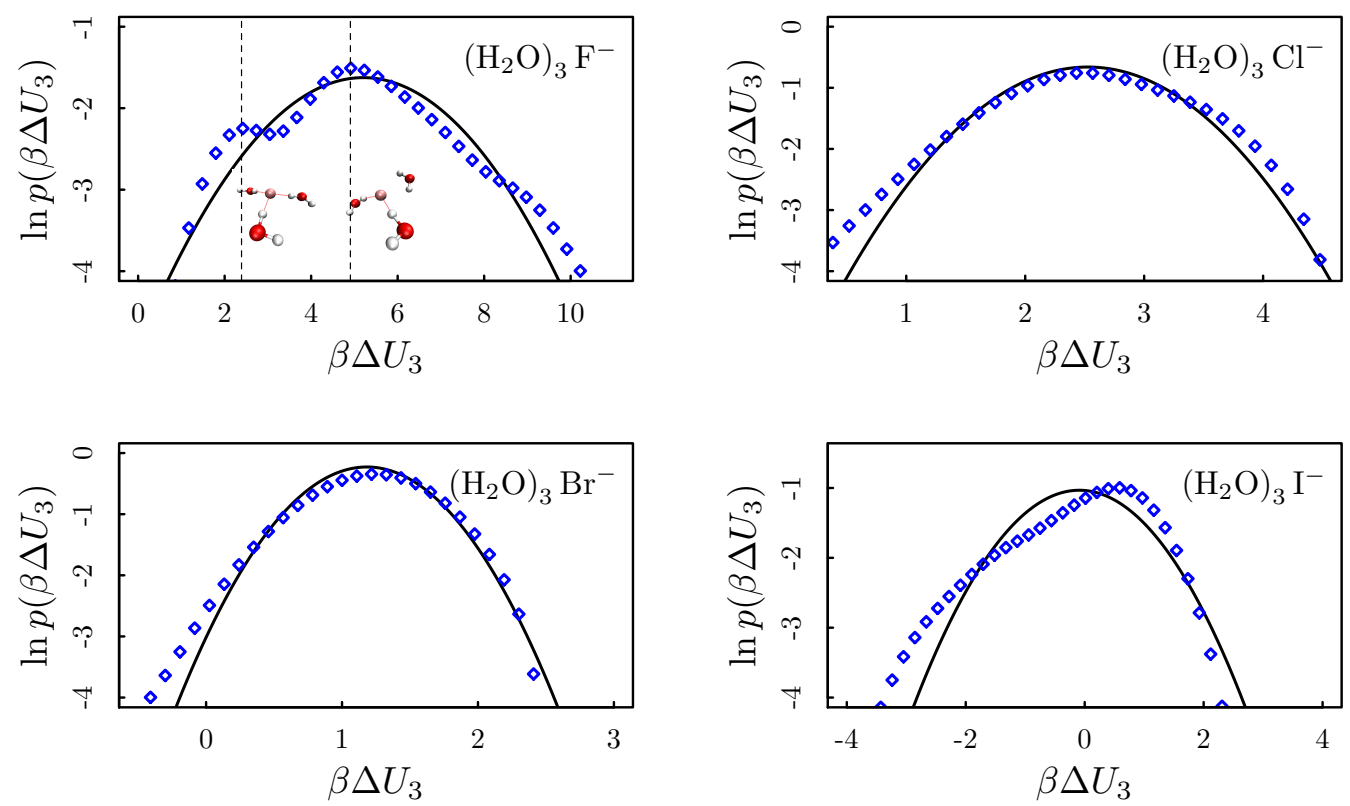

Figure 5. Distributions of $\beta \Delta U_{3}$ of Eq. (5) for halide-water clusters, observed during $10 \mathrm{ps}$ of $\mathrm{CP} 2 \mathrm{~K}$ trajectory after 5 ps aging. Split-shell geometries are not expected to be important for these $n=3$ cases (Figure 1). Nevertheless, $\mathrm{W}_{3} \mathrm{~F}^{-}$shows a multimodal distribution, the maxima located by dashed lines. The lower energy mode can be associated with the optimized structure (Figure 1) while the higher energy mode describes configurations that can flip a water molecule to offer a different $\mathrm{H}$ atom for coordination with the ion. The solid lines are the Gaussian model distribution with the sample mean and variance. These $\beta \Delta U_{3}$ reflect contributions from crowding of ligands, mostly unfavorable here. The estimated mean for $\mathrm{W}_{3} \mathrm{I}^{-}$is near zero, consistent with the realized performance of the harmonic approximation of that case (Figure 1). 
Laboratory Institutional Computing Program, supported by the U. S. Department of Energy National Nuclear Security Administration under Contract No. 89233218CNA000001. We thank Robert P. Currier and Katie A. Maerzke for their assistance.

Acknowledgments: This work was performed, in part, at the Center for Integrated Nanotechnologies, an Office of Science User Facility operated for the U.S. Department of Energy (DOE) Office of Science. Sandia National Laboratories is a multimission laboratory managed and operated by National Technology \& Engineering Solutions of Sandia, LLC, a wholly owned subsidiary of Honeywell International, Inc., for the U.S. DOE's National Nuclear Security Administration under contract DE-NA-0003525. This work was carried out in part at Oak Ridge National Laboratory, managed by UT-Battelle, LLC, for the US Department of Energy under contract DE-AC0500OR22725. The views expressed in the article do not necessarily represent the views of the US DOE or the United States Government.

Conflicts of Interest: The authors declare no conflict of interest. The funders had no role in the design of the study; in the collection, analyses, or interpretation of data; in the writing of the manuscript, or in the decision to publish the results.
Abbreviations
QCT Quasi-chemical theory
AIMD ab initio molecular dynamics

The following abbreviations are used in this manuscript:

\section{References}

1. Hofmeister, F. Zur Lehre von der Wirkung der Salze. Naunyn-Schmiedeberg's Archives of Pharmacology 1888, 24, 247-260.

2. Kunz, W.; Henle, J.; Ninham, B.W. 'Zur Lehre von der Wirkung der Salze' (about the science of the effect of salts): Franz Hofmeister's historical papers. Curr. Opin. Colloid Interface Sci. 2004, 9, 19-37.

3. Zhang, Y.; Cremer, P. Chemistry of Hofmeister anions and osmolytes. Annu. Rev. Phys. Chem. 2010, 61, 63-83.

4. Kunz, W. Specific ion effects, evidences. In Encyclopedia of Applied Electrochemistry; Kreysa, G.; Ota, K.i.; Savinell, R.F., Eds.; Springer New York: New York, NY, 2014; pp. 2050-2053. doi:10.1007/978-1-4419-6996-5_27.

5. Okur, H.I.; Hladílková, J.; Rembert, K.B.; Cho, Y.; Heyda, J.; Dzubiella, J.; Cremer, P.S.; Jungwirth, P. Beyond the Hofmeister series: Ion-specific effects on proteins and their biological functions. J. Phys. Chem. B 2017, 121, 1997-2014, [https://doi.org/10.1021/acs.jpcb.6b10797]. PMID: 28094985, doi:10.1021/acs.jpcb.6b10797.

6. Mazzini, V.; Liu, G.; Craig, V.S.J. Probing the Hofmeister series beyond water: Specific-ion effects in non-aqueous solvents. J. Chem. Phys. 2018, 148, 222805, [https://doi.org/10.1063/1.5017278]. doi:10.1063/1.5017278.

7. Hribar-Lee, B.; Vlachy, V.; Dill, K. Modeling Hofmeister effects. Acta Chim Slov. 2009, 56, 196-202.

8. Horinek, D. Specific Ion Effects, Theory. In Encyclopedia of Applied Electrochemistry; Kreysa, G.; Ota, K.i.; Savinell, R.F., Eds.; Springer New York: New York, NY, 2014; pp. 2050-2053. doi:10.1007/978-1-4419-6996-5_27.

9. Pollard, T.P.; Beck, T.L. Toward a quantitative theory of Hofmeister phenomena: From quantum effects to thermodynamics. Curr. Opin. In. 2016, 23, 110-118. doi:https:/ /doi.org/10.1016/j.cocis.2016.06.015.

10. Varma, S.; Rogers, D.M.; Pratt, L.R.; Rempe, S.B. Design principles for $\mathrm{K}^{+}$selectivity in membrane transport. J. Gen. Physiol. 2011, 137, 479-488.

11. Stevens, M.J.; Rempe, S.L.B. Ion-specific effects in carboxylate binding sites. J. Phys. Chem. B 2016, 120, 12519-12530, [https://doi.org/10.1021/acs.jpcb.6b10641]. PMID: 27973817, doi:10.1021/acs.jpcb.6b10641.

12. Chaudhari, M.I.; Vanegas, J.M.; Pratt, L.R.; Muralidharan, A.; Rempe, S.B. Hydration Mimicry by Membrane Ion Channels. Ann. Rev. Phys. Chem. 2019, [1912.04102].

13. Pratt, L.R.; Rempe, S.B. Quasi-chemical theory and implicit solvent models for simulations. AIP Conference Proceedings. American Institute of Physics, 1999, Vol. 492, pp. 172-201.

14. Asthagiri, D.; Dixit, P.; Merchant, S.; Paulaitis, M.; Pratt, L.; Rempe, S.; Varma, S. Ion selectivity from local configurations of ligands in solutions and ion channels. Chem. Phys, Letts. 2010, 485, 1-7. doi:10.1016/j.cplett.2009.12.013.

15. Muralidharan, A.; Pratt, L.R.; Chaudhari, M.I.; Rempe, S.B. Quasi-chemical theory for anion hydration and specific ion effects: $\mathrm{Cl}^{-}$(aq) vs. $\mathrm{F}^{-}$(aq). Chem. Phys. Letts.: X 2019, 4, 100037. doi:10.1016/j.cpletx.2019.100037.

16. Frenkel, J. Statistical Theory of Condensation Phenomena. J. Chem. Phys. 1939, 7, 200-201. doi:10.1063/1.1750413.

17. Band, W. Dissociation Treatment of Condensing Systems. J. Chem. Phys. 1939, 7, 324-326. doi:10.1063/1.1750444.

18. Band, W. Dissociation Treatment of Condensing Systems. II. J. Chem. Phys. 1939, 7, 927-931. doi:10.1063/1.1750346.

19. Stillinger, F.H. Rigorous Basis of the Frenkel-Band Theory of Association Equilibrium. J. Chem. Phys. 1963, 38, 1486-1494. doi:10.1063/1.1776907. 
20. Stillinger, F.H. Physical Clusters, Surface Tension, and Critical Phenomena. J. Chem. Phys. 1967, 47, $2513-2533$. doi:10.1063/1.1712264.

21. Chandler, D.; Pratt, L.R. Statistical mechanics of chemical equilibria and intramolecular structures of nonrigid molecules in condensed phases. J. Chem. Phys. 1976, 65, 2925-2940. doi:10.1063/1.433529.

22. Lockett, A.M. A theory of homogeneous condensation from small nuclei. I. Modified Mayer theory of physical clusters. J. Chem. Phys. 1980, 72, 4822-4831. doi:10.1063/1.439820.

23. Pratt, L.R.; LaViolette, R.A. Quasi-chemical theories of associated liquids. Mol. Phys. 1998, 94, 909-915, [physics/9803018]. doi:10.1080/002689798167485.

24. Fisher, M.E. The Theory of Condensation and the Critical Point. Physics 1967, 3, 255 - 283.

25. Pratt, L.R.; Asthagiri, D. Potential distribution methods and free energy models of molecular solutions. In Free Energy Calculations; Springer, 2007; pp. 323-351.

26. Gadre, S.R.; Yeole, S.D.; Sahu, N. Quantum Chemical Investigations on Molecular Clusters. Chem. Rev. 2014, 114, 12132-12173. doi:10.1021/cr4006632.

27. Rempe, S.; Jonsson, H. A computational exercise illustrating molecular vibrations and normal modes. Chem. Educat. 1998, 3, 1-17.

28. Zhang, J.; Glezakou, V.A. Global optimization of chemical cluster structures: Methods, applications, and challenges. Int. J. Quant. Chem. 2021, 121, e26553, [https:/ / onlinelibrary.wiley.com/doi/pdf/10.1002/qua.26553]. doi:https://doi.org/10.1002/qua.26553.

29. Jesus, W.; Prudente, F.; Marques, J.; Pereira, F. Modeling microsolvation clusters with electronic-structure calculations guided by analytical potentials and predictive machine learning techniques. Phys. Chem. Chem. Phys. 2021, 23, 1738-1749.

30. Rogers, D.M.; Rempe, S.B. Probing the thermodynamics of competitive ion binding using minimum energy structures. J. Phys. Chem. B 2011, 115, 9116-9129, [https:/ / doi.org/10.1021/jp2012864]. PMID: 21721551, doi:10.1021/jp2012864.

31. Rogers, D.M.; Jiao, D.; Pratt, L.R.; Rempe, S.B. Chapter Four - Structural Models and Molecular Thermodynamics of Hydration of Ions and Small Molecules. In Annual Reports in Computational Chemistry; Wheeler, R.A., Ed.; Elsevier, 2012; Vol. 8, Annu. Rep. Comput. Chem., pp. 71-127. doi:https://doi.org/10.1016/B978-0-444-59440-2.00004-1.

32. Sabo, D.; Varma, S.; Martin, M.G.; Rempe, S.B. Studies of the thermodynamic properties of hydrogen gas in bulk water. J. Phys. Chem. B 2008, 112, 867-876, [https:/ / doi.org/10.1021/jp075459v]. PMID: 18154326, doi:10.1021/jp075459v.

33. Perera, L.; Berkowitz, M.L. Structure and dynamics of $\mathrm{Cl}^{-}\left(\mathrm{H}_{2} \mathrm{O}\right)_{n}$ clusters: The effect of the polarizability and the charge of the ion. J. Chem. Phys. 1992, 96, 8288 - 8294. doi:10.1063/1.462332.

34. Perera, L.; Berkowitz, M.L. Stabilization energies of $\mathrm{Cl}^{-}, \mathrm{Br}^{-}$, and $\mathrm{I}^{-}$ions in water clusters. J. Chem. Phys. 1993, $99,4222-4224$. doi:10.1063/1.466096.

35. Perera, L.; Berkowitz, M.L. Structures of $\mathrm{Cl}^{-}\left(\mathrm{H}_{2} \mathrm{O}\right)_{n}$ and $\mathrm{F}^{-}\left(\mathrm{H}_{2} \mathrm{O}\right)_{n}(n=2,3, \ldots, 15)$ clusters. Molecular dynamics computer simulations. J. Chem. Phys. 1994, 100, 3085 - 3093. doi:10.1063/1.466450.

36. Sremaniak, L.S.; Perera, L.; Berkowitz, M.L. Enthalpies of formation and stabilization energies of $\mathrm{Br}^{-}\left(\mathrm{H}_{2} \mathrm{O}\right)_{n}$. Chem. Phys. Letts. 1994, pp. $377-382$.

37. Combariza, J.E.; Kestner, N.R.; Jortner, J. Microscopic solvation of anions in water clusters. Chem. Phys. Letts. 1993, 203, 423-428. doi:10.1016/0009-2614(93)85592-c.

38. Combariza, J.E.; Kestner, N.R. Microscopic Study of Fluoride-Water Clusters. J. Phys. Chem. 1994, 98, $3513-3517$. doi:10.1021/j100064a038.

39. Combariza, J.E.; Kestner, N.R.; Jortner, J. Energy-structure relationships for microscopic solvation of anions in water clusters. J. Chem. Phys. 1994, 100, 2851-2864. doi:10.1063/1.467231.

40. Combariza, J.E.; Kestner, N.R.; Jortner, J. Surface and interior states of iodide-water clusters. Chem. Phys. Letts. 1994, 221, 156-160. doi:10.1016/0009-2614(94)87032-2.

41. Combariza, J.E.; Kestner, N.R. Density Functional Study of Short-Range Interaction Forces between Ions and Water Molecules. J. Phys. Chem. 1995, 99, 2717-2723. doi:10.1021/j100009a030.

42. Cheshnovsky, O.; Giniger, R.; Markovich, G.; Makov, G.; Nitzan, A.; Jortner, J. Surface and interior anion solvation in water clusters. J. Chim. Phys. 1995, 92, 397-408. doi:10.1051/jcp/1995920397.

43. Choi, J.H.; Kuwata, K.T.; Cao, Y.B.; Okumura, M. Vibrational Spectroscopy of the $\mathrm{Cl}^{-}\left(\mathrm{H}_{2} \mathrm{O}\right)_{n}$ Anionic Clusters, $n=1-5$. J. Phys. Chem. A 1998, 102, 503-507.

44. Basdogan, Y.; Groenenboom, M.C.; Henderson, E.; De, S.; Rempe, S.B.; Keith, J.A. Machine learning-guided approach for studying solvation environments. J. Chem. Theory Comput. 2020, 16, 633-642, [https://doi.org/10.1021/acs.jctc.9b00605]. PMID: 31809056, doi:10.1021/acs.jctc.9b00605.

45. Tissandier, M.D.; Cowen, K.A.; Feng, W.Y.; Gundlach, E.; Cohen, M.H.; Earhart, A.D.; Coe, J.V.; Tuttle, T.R. The proton's absolute aqueous enthalpy and Gibbs free energy of solvation from cluster-ion solvation data. J. Phys. Chem. A 1998, 102, 7787-7794.

46. Rogers, D.M.; Beck, T.L. Quasichemical and structural analysis of polarizable anion hydration. J. Chem. Phys. 2010, 132, 01450513. doi:10.1063/1.3280816.

47. Perdew, J.P.; Burke, K.; Ernzerhof, M. Errata: Generalized Gradient Approximation Made Simple. Phys. Rev. Lett 1996, $78,1396$.

48. Rempe, S.B.; Pratt, L.R.; Hummer, G.; Kress, J.D.; Martin, R.L.; Redondo, A. The hydration number of Li+ in liquid water. J. Am. Chem. Soc. 2000, 122, 966-967, [https://doi.org/10.1021/ja9924750]. doi:10.1021/ja9924750.

49. Rempe, S.B.; Pratt, L.R. The hydration number of $\mathrm{Na}^{+}$in liquid water. Fluid Ph. Equilibria 2001, 183-184, 121-132. Proceedings of the fourteenth symposium on thermophysical properties, doi:https://doi.org/10.1016/S0378-3812(01)00426-5. 
50. Rempe, S.B.; Asthagiri, D.; Pratt, L.R. Inner shell definition and absolute hydration free energy of $\mathrm{K}^{+}$(aq) on the basis of quasi-chemical theory and ab initio molecular dynamics. Phys. Chem. Chem. Phys. 2004, 6, 1966-1969.

51. Sabo, D.; Jiao, D.; Varma, S.; Pratt, L.R.; Rempe, S.B. Case study of $\mathrm{Rb}^{+}$(aq), quasi-chemical theory of ion hydration, and the no split occupancies rule. Annu. Rep. Prog. Chem., Sect. C: Phys. Chem. 2013, 109, 266-278. doi:10.1039/C3PC90009F.

52. Chaudhari, M.I.; Soniat, M.; Rempe, S.B. Octa-Coordination and the aqueous Ba ${ }^{2+}$ iIon. J. Phys. Chem. B 2015, 119, 8746-8753, [https://doi.org/10.1021/acs.jpcb.5b03050]. PMID: 26085171, doi:10.1021/acs.jpcb.5b03050.

53. Chaudhari, M.I.; Rempe, S.B. Strontium and barium in aqueous solution and a potassium channel binding site. J. Chem. Phys. 2018, 148, 222831, [https:/ / doi.org/10.1063/1.5023130]. doi:10.1063/1.5023130.

54. Soniat, M.; Rogers, D.M.; Rempe, S.B. Dispersion- and exchange-corrected density functional theory for sodium ion hydration. J. Chem. Theory Comput. 2015, 11, 2958-2967, [https://doi.org/10.1021/acs.jctc.5b00357]. PMID: 26575733, doi:10.1021/acs.jctc.5b00357.

55. VandeVondele, J.; Krack, M.; Mohamed, F.; Parrinello, M.; Chassaing, T.; Hutter, J. Quickstep: Fast and accurate density functional calculations using a mixed Gaussian and plane waves approach. Computer Physics Communications 2005, 167, $103-128$.

56. Kühne, T.D.; Iannuzzi, M.; Ben, M.D.; Rybkin, V.V.; Seewald, P.; Stein, F.; Laino, T.; Khaliullin, R.Z.; Schütt, O.; Schiffmann, F.; Golze, D.; Wilhelm, J.; Chulkov, S.; Bani-Hashemian, M.H.; Weber, V.; Borštnik, U.; Taillefumier, M.; Jakobovits, A.S.; Lazzaro, A.; Pabst, H.; Müller, T.; Schade, R.; Guidon, M.; Andermatt, S.; Holmberg, N.; Schenter, G.K.; Hehn, A.; Bussy, A.; Belleflamme, F.; Tabacchi, G.; Glöß, A.; Lass, M.; Bethune, I.; Mundy, C.J.; Plessl, C.; Watkins, M.; VandeVondele, J.; Krack, M.; Hutter, J. CP2K: An electronic structure and molecular dynamics software package - Quickstep: Efficient and accurate electronic structure calculations. J. Chem. Phys. 2020, 152, 194103, [2003.03868]. doi:10.1063/5.0007045.

57. Zhao, Y.; Truhlar, D.G. A new local density functional for main-group thermochemistry, transition metal bonding, thermochemical kinetics, and noncovalent interactions. J. Chem. Phys. 2006, 125, 194101, [https://doi.org/10.1063/1.2370993]. doi:10.1063/1.2370993.

58. Goedecker, S.; Teter, M.; Hutter, J. Separable Dual-Space Gaussian Pseudopotentials. Phys. Rev. B 1996, $54,1703$.

59. Lippert, G.; Hutter, J.; Parrinello, M. The Gaussian and augmented-plane-wave density functional method for ab initio molecular dynamics simulations. Theo. Chem. Accts. 1999, 103, 124-140.

60. VandeVondele, J.; Hutter, J. Gaussian basis sets for accurate calculations on molecular systems in gas and condensed phases. J. Chem. Phys. 2007, 127, 114105.

61. Kitao, O.; Nakai, H.; Vreven, T.; Montgomery, J.; Peralta, J.; Ogliaro, F.; Bearpark, M.; Heyd, J.; Brothers, E.; Kudin, K.; others. Gaussian 09, revision A. 1. Gaussian Inc.: Wallingford, CT 2009.

62. Jain, R.; Ahuja, B.; Sharma, B. Density-Functional Thermochemistry. III. The Role of Exact Exchange. Indian J. Pure E Appl. Phys. 2004, 42, 43-48.

63. Lee, C.; Yang, W.; Parr, R.G. Development of the Colle-Salvetti correlation-energy formula into a functional of the electron density. Phys. Rev. B 1988, 37, 785.

64. Perdew, J.P.; Burke, K.; Ernzerhof, M. Generalized Gradient Approximation Made Simple. Phys. Rev. Lett. 1996, 77, 3865-3868. doi:10.1103/PhysRevLett.77.3865.

65. Perdew, J.P.; Burke, K.; Ernzerhof, M. Generalized Gradient Approximation Made Simple [Phys. Rev. Lett. 77, 3865 (1996)]. Phys. Rev. Lett. 1997, 78, 1396-1396. doi:10.1103/PhysRevLett.78.1396.

66. Kendall, R.A.; Dunning Jr, T.H.; Harrison, R.J. Electron affinities of the first-row atoms revisited. Systematic basis sets and wave functions. J. Chem. Phys. 1992, 96, 6796-6806.

67. Woon, D.E.; Dunning Jr, T.H. Gaussian basis sets for use in correlated molecular calculations. III. The atoms aluminum through argon. J. Chem. Phys. 1993, 98, 1358-1371.

68. Weigend, F.; Ahlrichs, R. Balanced basis sets of split valence, triple zeta valence and quadruple zeta valence quality for $H$ to Rn: Design and assessment of accuracy. Phys. Chem. Chem. Phys. 2005, 7, 3297-3305.

69. Weigend, F.; Ahlrichs, R. Balanced basis sets of split valence, triple zeta valence and quadruple zeta valence quality for $H$ to Rn: Design and assessment of accuracy. Phys. Chem. Chem. Phys. 2005, 7, 3297-3305.

70. Muralidharan, A.; Pratt, L.R.; Chaudhari, M.I.; Rempe, S.B. Quasi-Chemical Theory with Cluster Sampling from Ab Initio Molecular Dynamics: Fluoride $\left(\mathrm{F}^{-}\right)$Anion Hydration. J. Phys. Chem. A 2018, pp. acs.jpca.8b08474 - 7. doi:10.1021/acs.jpca.8b08474.

71. Rappoport, D.; Furche, F. Property-optimized Gaussian basis sets for molecular response calculations. J. Chem. Phys. 2010, $133,134105$.

72. Smith, D.G.; Burns, L.A.; Simmonett, A.C.; Parrish, R.M.; Schieber, M.C.; Galvelis, R.; Kraus, P.; Kruse, H.; Di Remigio, R.; Alenaizan, A.; others. PSI4 1.4: Open-source software for high-throughput quantum chemistry. The Journal of chemical physics 2020, 152, 184108.

73. Beck, T.E.; Paulaitis, M.E.; Pratt, L.R. Potential distribution theorem and statistical thermodynamics of molecular solutions; Cambridge University Press: New York, 2006. 